\title{
Diffraction of Light by Finite Amplitude Ultrasonic Waves
}

\author{
L. ADLER*
}

Ohio State University/Adler Consultants Inc., 1560 Gulf Blvd. \#1002, Clearwater, Florida, USA

In the late 1950s the Ultrasonic Group at Michigan State University introduced light diffraction to study distortion of ultrasonic waves in liquids under the direction of Prof. Egon Hiedemann. In this paper some results of these studies will be presented with detailed description of the author's measurements of $B / A$. The rate at which the harmonics are developed (the wave distortion is an indication of the harmonics present) during the propagation of the initially sinusoidal ultrasonic wave depends on the nonlinearity of the medium. The light which is diffracted by the distorted wave results in asymmetric pattern contrary to the Raman-Nath theoretical prediction. From the light intensity measurements due to the generated second harmonics — filtered out by a metal plate - the nonlinearity parameter $B / A$ was determined. Developments in studying finite amplitude standing ultrasonic waves in a liquid filled cavity will be also discussed. Using light diffraction measurements it was observed that above a threshold amplitude, fractional harmonics of the driver transducer are also generated in addition to the generated harmonics. It was recently observed that above a second threshold value of the driver amplitude the system undergoes chaotic behavior. Further increase of the driver amplitude returns the system from chaos to stable oscillation.

DOI: $10.12693 /$ APhysPolA.127.5

PACS: 43.25.Ba, 43.35.Sx

\section{Introduction and background}

\subsection{Diffraction of light by ultrasonic waves}

In 1932, Debye and Sears [1] in the USA and Lucas and Biquard [2] in France independently observed that when a monochromatic light beam propagates perpendicularly through an ultrasonic beam, the light will diffract into several orders. A theoretical model was suggested by Sir Raman and Nath [3] (it is known today as the RamanNath theory). They proposed that the ultrasonic wave behaves like a diffraction grating for the light which will produce the diffraction orders. Starting with the electromagnetic wave equation and introducing a variable refractive index for the light, they were able to predict the intensity of each order as well as the positions of the orders. The intensity of the diffracted light in the $\mathrm{m} n$-th order is proportional to the square of the $\mathrm{m} n$-th order Bessel function normalized to the incident light intensity as given by Eq. (1),

$$
I_{n}=J_{n}^{2}(v) \text {. }
$$

$J_{n}$ is the $\mathrm{m} n$-th order Bessel function with the argument $v$, which is called the Raman-Nath parameter related to the pressure of the ultrasonic wave

$$
v=2 \pi \mu a / \lambda,
$$

where $a$ is the width of the sound field, $\lambda$ is the wavelength of the light and $\mu$ is the variation of the refractive index of the liquid due to the density variation caused by the ultrasonic pressure. The angle $\Theta$ of the diffracted light is given

$$
\sin (\Theta)=n \lambda / \lambda^{*}
$$

where $\lambda^{*}$ is the wavelength of the sound.

\footnotetext{
*e-mail: Ladler1@aol.com
}

\subsection{Diffraction of light}

by finite amplitude ultrasonic waves

The intensity distribution of the diffracted light is symmetrical i.e. the intensity of the first diffracted light to the right from the incident light is the same as to the left, because it is the square of the Bessel functions. The theory of Raman and Nath was in good agreement with the experimental results for low intensity ultrasound i.e. for infinitesimally small ultrasonic amplitudes. Zankel [4] observed asymmetry in the diffraction pattern. It was demonstrated that the major effect of the asymmetry is due to the large amplitude of the ultrasonic waves or the so-called finite amplitude in contrast with the infinitesimal amplitude. As a sinusoidal wave propagates with finite amplitude the ultrasonic waveform distorts thus causing the asymmetry in the light diffraction pattern. The asymmetry in the first orders (the difference in the negative and positive order) of the measured light intensity increases with distance as shown in Fig. 1, illustrating that the wave distortion increases with distance. The asymmetry in the diffraction pattern also increases with the increase of fundamental pressure as illustrated in a photograph in Fig. 2, which was taken by Breazeale [5].

The asymmetry in the diffraction pattern is increasing with increasing distance of the ultrasound and with increasing sound intensity as illustrated in Fig. 2. The distortion of the ultrasonic waveform will be explained after a brief review of nonlinear acoustics which is described briefly in the next section.

\subsection{Nonlinear acoustics in fluids}

In general, a sound beam changes its wave shape as it propagates in a fluid (as well as in solids) whose pressure density relationship does not obey Hooke's law. This 

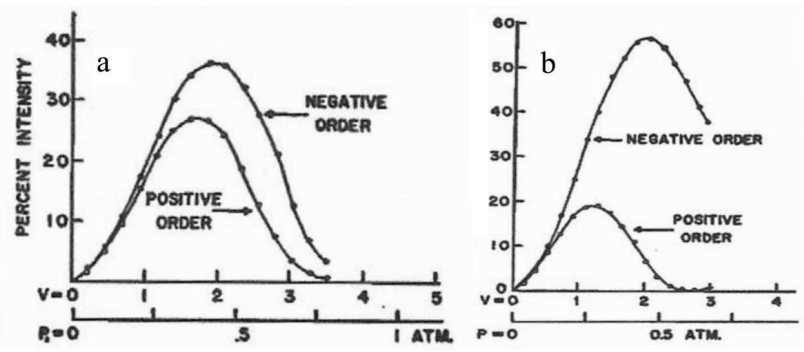

Fig. 1. Intensity of light measured in the first order for a $4 \mathrm{MHz}$ ultrasonic wave in water as function of the pressure at a distance from the source (a) at $10 \mathrm{~cm}$ and (b) at $50 \mathrm{~cm}$. Reproduced with permission from Ref. [4]. Copyright by Acoustical Society of America.

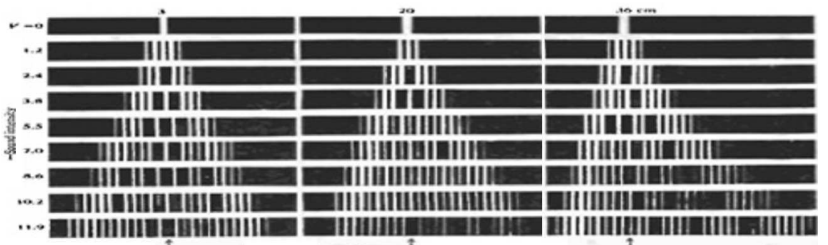

Fig. 2. Light diffraction by ultrasonic waves in water with frequency of $1.76 \mathrm{MHz}$ at 3,20 , and at $36 \mathrm{~cm}$. Reproduced with permission from Ref. [5]. Copyright by Acoustical Society of America.

means that harmonics are generated as the wave progresses. For infinitesimal amplitudes the change in wave shape is so small that it can be neglected, however, it has to be considered for finite amplitude waves. The distortion of a finite amplitude wave is due to the nonlinear property of the medium in which the wave propagates.

Fox and Wallace [6] in 1959 assumed a power series relation between pressure and density

$$
P=P_{0}+A\left(\rho-\rho_{0}\right) / \rho+B / 2\left(\rho-\rho_{0} / \rho_{0}\right)^{2},
$$

where $P$ and $\rho$ are the instantaneous pressure and density of the liquid, respectively, $P_{0}$ and $\rho_{0}$ are the pressure and density of the undisturbed liquid and $B / A$ is called the nonlinearity parameter for liquids. They derived a nonlinear wave equation for liquids, given in Eq. (5) for a one-dimensional dissipationless case, for the particle displacement $\xi$,

$$
\partial^{2} \xi / \partial t^{2}=c^{2}\left(\partial^{2} \xi / \partial x^{2}\right) /(1+\partial \xi / \partial x)^{(B / A+2)},
$$

where $\xi$ is the particle displacement and $c$ is the velocity of sound in the liquid.

For infinitesimal amplitude waves the value of $\partial \xi / \partial x$ is much less than 1 and it can be neglected and Eq. (5) reduces to the linear wave equation as expected. FubiniGhiron [7] in 1935 used an analytical method to obtain an exact solution of the nonlinear wave equation, the paper which was published in Italian in Alta Frequenza was not discovered before the same solutions were obtained independently by Hargrove [8] (at Michigan State University) and Keck and Bayer [9] (Brown University) in 1960.

The solutions of Eq. (5) for the pressure amplitudes $P_{n}$ of the $\mathrm{m} n$-th harmonic of the distorted wave are given as

$$
P_{n}(k)=\left(2 P_{1}(0) / n k\right) J_{n}(n k),
$$

where $J_{n}$ is the $\mathrm{m} n$-th order Bessel function, $k=x / L$, the ratio of the propagation distance $x$ to the discontinuity distance $L$, that is the distance in a dissipationless liquid where the distorted wave slope will become infinite. The value of $L$ is given by

$$
L=c^{3} \rho_{0}\left\{2[B /(2 A)+1] P_{1}(0) f\right\}^{-1},
$$

where $P_{1}(0)$ is the peak acoustic pressure amplitude of the initial sinusoidal wave with frequency $f$.

\subsection{Determination of the nonlinearity parameter $B / A$ for liquids}

One can calculate an expression for the amplitude of the second harmonic at any distance $x$ from the source by expanding the Bessel function in a power series and dropping higher order terms

$$
P_{2}(x)=\pi / 2\left[(B / A+2) / \rho_{0} c^{3}\right]\left(x f P 1(0)^{2} .\right.
$$

From Eq. (8), the nonlinearity parameter $B / A$ is given as

$$
B / A=2 c^{3} \rho_{0} / \pi f\left[P_{2}(x) / x P_{1}(0)^{2}-2\right] .
$$

Curves representing $P_{2}(x) / P_{1}(0)^{2}$ for a given medium, temperature and frequency and for various distances and initial pressures should extrapolate with zero slope to a common point for $x P_{1}(0)=0$. The nonlinearity parameter $B / A$ may then be determined from

$$
B / A=\left\{\left(2 c^{3} \rho_{0} / \pi f\right)\right\}\left[P_{2}(x) / x P_{1}(0)^{2}\right]_{x P 1(0)=0}-2 .
$$

As can be seen from Eq. (10) the amplitude of the second harmonic $P_{2}(x)$ and the amplitude of the fundamental $P_{1}(0)$ need to be measured to obtain the value of $B / A$ for a given liquid and at a given frequency of the initial ultrasonic wave.

\subsection{Experimental arrangement and procedure to determine $B / A$}

The experimental arrangement is shown schematically in Fig. 3. Light from the $\mathrm{Hg}$ vapor lamp $\mathrm{S}$ is condensed by lens $\mathrm{L}_{1}$ onto the source slit $\mathrm{SL}_{1}$. The lens $\mathrm{L}_{2}$ is adjusted by autocollimation to render the light parallel. The collimated light beam passes through a specially designed tank, and lens $\mathrm{L}_{3}$ produces an image of the source slit $\mathrm{SL}_{1}$ in the plane of the entrance slit $\mathrm{SL}_{2}$ of the photomultiplier microphotometer P. A filter which passes the $5461 \AA$ $\mathrm{Hg}$ line is located inside the photomultiplier. The transducer $\mathrm{Q}$ is air-backed by $1 \times 1 \mathrm{in}^{2}$ quartz excited at its fundamental frequency. The acoustic filter $\mathrm{F}$ is a stainless steel plate with a $1 \mathrm{~mm}$ thickness.

When a light passes through an ultrasonic wave in the liquid, various orders of diffraction are observed in the plane of the photomultiplier entrance slit.

The peak change of refractive index $\mu$ is related to the peak sound pressure amplitude. From measurements of light intensity in the diffracted orders $v$ may be determined from Eq. (1). To determine the pressure amplitude $P$ from $v$ the relationship between the change of index refraction $\mu$ and change in pressure $P$ must be known. In this work we used a formula obtained by Eykman [11] in 1895: 


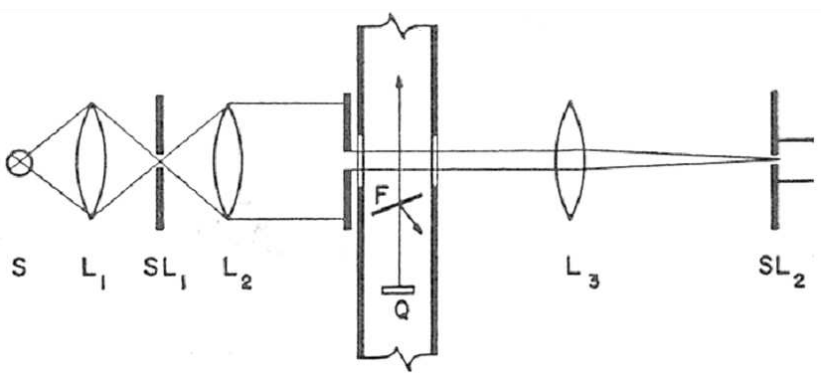

Fig. 3. Schematic diagram of the experimental arrangement. Reproduced with permission from Ref. [10]. Copyright by Acoustical Society of America.

$$
\mu=\left[\left(\mu_{0}-1\right)\left(\mu_{0}+1.4 \mu_{0}+0.4\right) /\left(\mu_{0}^{2}+0.8 \mu_{0}+1\right)\right] P,
$$

where $\mu_{0}$ is the refractive index of the undisturbed medium and $P$ is the change of pressure.

Using Eq. (11) we obtain the relationship between $P$ and $v$ :

$$
P=0.56 v / a[\mathrm{~atm}]
$$

for water and

$$
P=0.25 v / a[\mathrm{~atm}]
$$

for $m$-xylene, where $a$ is in $\mathrm{cm}$.

Determinations of $P_{1}(0)$ were made from diffraction order light intensities for small sound amplitudes and near the transducer where the waveform is undistorted (a necessary condition to use the Raman-Nath theory, Eq. (1)). It was then assumed that the voltage across the transducer and $P_{1}(0)$ are linearly related, values of $P_{1}(0)$ were obtained from the voltage across the transducer.

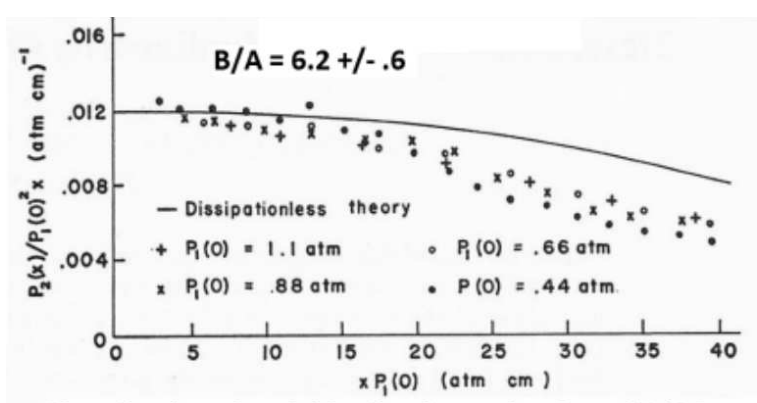

Plot of reduced variables for determination of $B / A$ in water

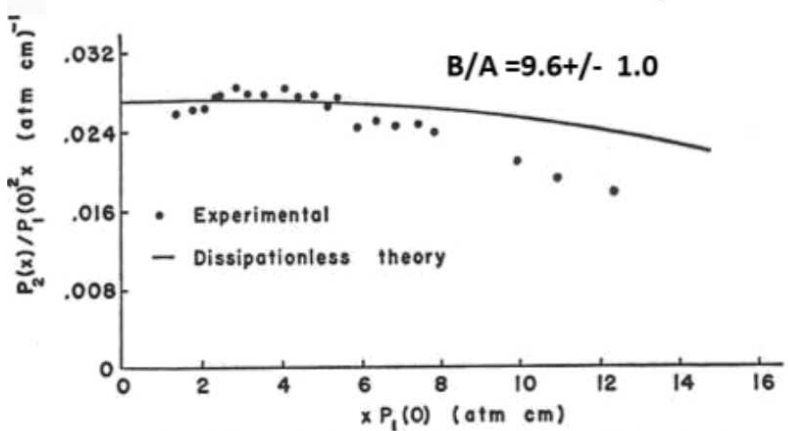

Plot of reduced variables for determination of $B / A$ for $m$-xylene

Fig. 4. Plots of the reduced variables for determination of $B / A$. Reproduced with permission from Ref. [10]. Copyright by Acoustical Society of America.

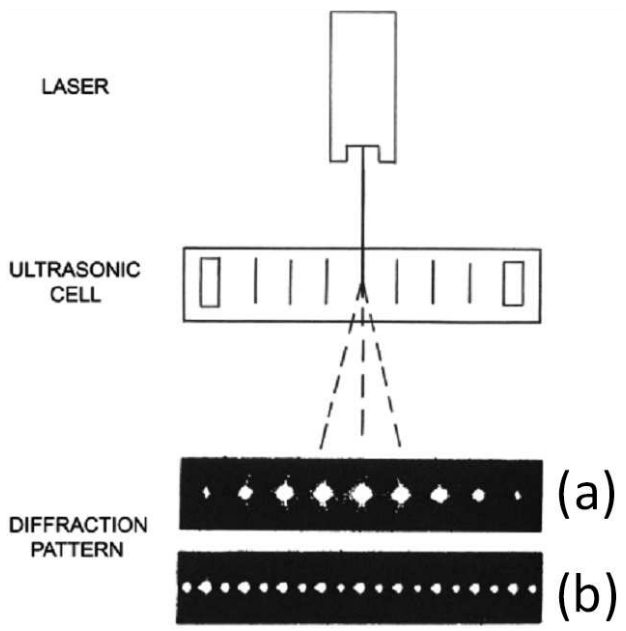

Fig. 5. Schematic diagram of the diffraction pattern produced by a laser beam diffracted by a finite amplitude ultrasonic standing wave in a water filled one dimensional cavity. Below the threshold (a) and above threshold (b).

For certain angles of incidence of the ultrasonic wave on the acoustic filter plate the fundamental component of the ultrasonic wave is reflected and the second harmonic is transmitted [5, 12]. Using a weak ultrasonic wave with fundamental frequency corresponding to the second harmonic frequency of the finite amplitude wave to be investigated, measurements of the second harmonic component of the finite amplitude ultrasonic waves were made in water and in $m$-xylene for a $3.0 \mathrm{MHz}$ fundamental frequency.

Figure 4 shows $P_{2}(x) / x\left(P_{1}(0) 2\right.$ vs. $x P_{1}(0)$ for water and Fig. 5 is a similar plot for $m$-xylene. From extrapolation of these data to $x P_{1}(0)=0$ and using Eq. (8):

$$
B / A=6.2
$$

for water and

$$
B / A=9.6
$$

for $m$-xylene. The estimated errors are within $10 \%$.

The value of $B / A$ for water is about $15 \%$ higher than what is reported later. One source of error of the optical measurement is due to the conversion from refractive index variation $\mu$ to the acoustic pressure $P$.

\section{Subharmonic generation in water-filled one-dimensional cavity}

While studying finite amplitude standing waves in a liquid filled cavity bounded at one end by a rigid reflector and the other end by a quartz transducer, extra orders in the diffraction pattern were observed $[13,14]$. The extra orders appear only above a certain threshold voltage of the driver transducer. In Fig. 5, the diffraction pattern is shown for below the threshold value of parametric excitation in Fig. 5a, and above the threshold in Fig. 5b.

The extra diffraction orders shown in Fig. 5b were obtained due to the subharmonics generated at approximately at half the frequency of the driver. 
The acoustical frequency spectrum shown in Fig. 6 corresponds to the optical diffraction pattern in Fig. 5b. It was taken by the spectrum analyzer. The driver frequency is called $2 f$, which is $4 \mathrm{MHz}$ in this case and the driver voltage is $100 \mathrm{~V}$.

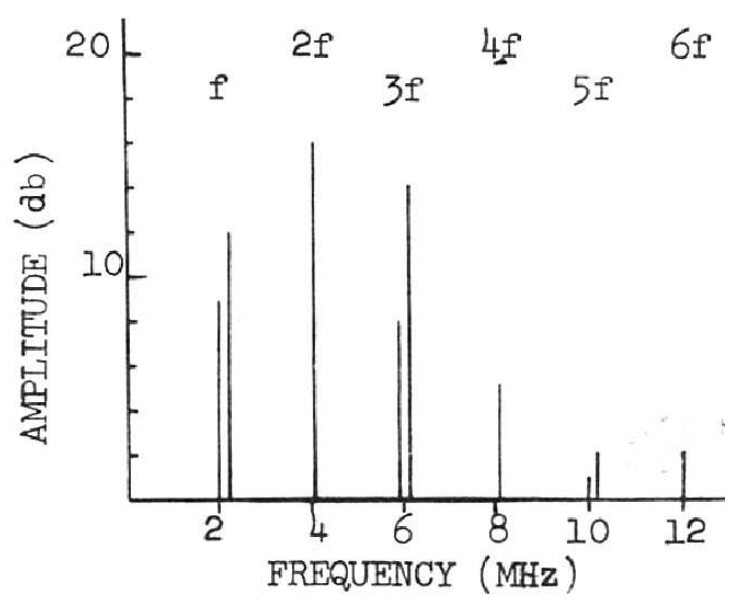

Fig. 6. Frequency spectrum at driver's voltage $100 \mathrm{~V}$.

The other frequencies are grouped as follows: (1) $4 f$, $6 f$ - these are the second and third harmonics of the driver. These components are present even below the threshold value of the parametric excitation and are due to the nonlinearity of the liquid. (2) The frequency pairs at $f, 3 f$, and $5 f$ i.e. about one half, three halves and five halves the driver frequency, are sometimes called more appropriately fractional harmonics. These components are affected by the amplitude of the driver and other parameters e.g. the liquid used, geometry e.g. the system etc. With increased amplitude of the driver, the complexity of the spectrum increases. It has been recognized only recently that the cascade of bifurcation leads essentially to chaos in the system, which will be discussed in the next section.

\section{Chaos and beyond in a liquid filled ultrasonic resonant system [15]}

As was pointed out in the previous section, discussing the mechanism of subharmonic generation, increase of the driver's amplitude produces a cascade of bifurcation. The problem was recently revisited with an improved experimental system shown in Fig. 7 .

It consists of an interferometer with optical precision controls used to adjust the positions of the piezo-electric transducer (1-10 $\mathrm{MHz}$ driven by a powerful amplifier) and a receiving transducer attached to an aligned reflector with lapped flat and parallel surfaces used to measure the generated frequency components in the cavity.

A visual assessment of the phenomena is obtained by passing laser light through the ultrasonic beam as indicated in Fig. 8. The laser light is diffracted into various orders $n$ at angles given by Eq. (3).

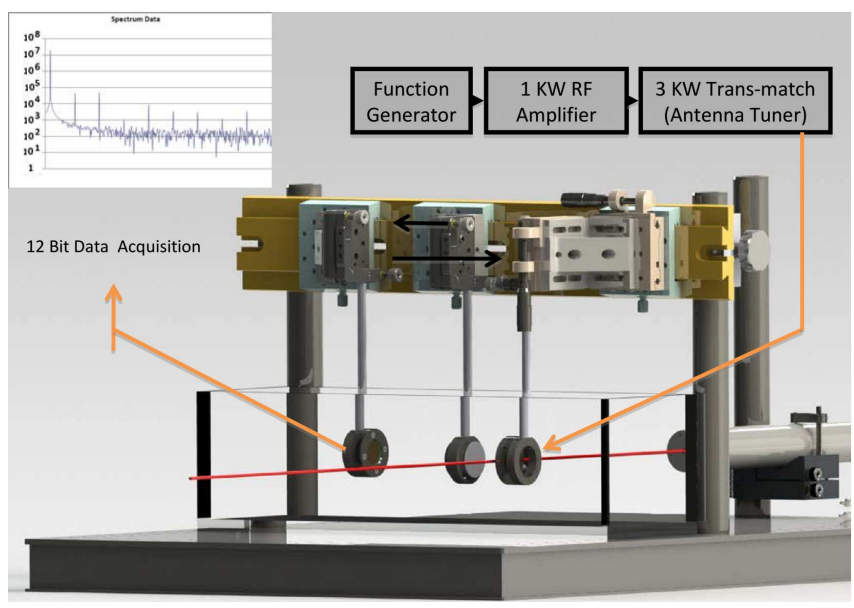

Fig. 7. Experimental system.

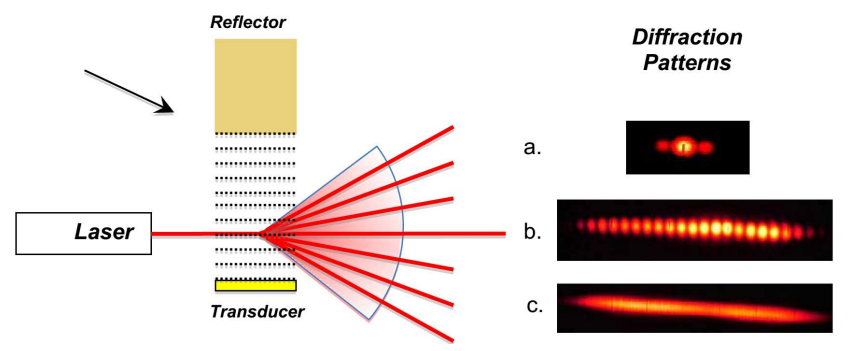

Fig. 8. Laser beam diffraction by ultrasonic wave: (a) linear region, (b) nonlinear region, (c) subharmonic region.

Figure 8 shows the diffraction patterns obtained for various transducer drive amplitudes (voltages): (a) low amplitude ultrasonic waves $(5 \mathrm{~V})$; (b) finite amplitude waves $(50 \mathrm{~V})$ resulting in an asymmetric diffraction pattern; and (c) parametric resonance $(150 \mathrm{~V})$ producing extra diffraction orders due to the generation of the subharmonics.

\subsection{Path to chaos}

A significantly higher transducer drive voltage (450 V) in the parametric resonance region leads to a cascade of bifurcations with increasing drive amplitudes that culminates in the generation of the chaotic pattern shown in Fig. 9a. Instead of distinct diffraction orders, the laser produces a smeared out image due to the chaotic oscillations. Further increases in the transducer drive voltage (to $500 \mathrm{~V}$ ) leads to a second region of stability following the region of chaotic instability. The diffraction pattern in the second region of stability is shown in Fig. $9 \mathrm{~b}$. The pattern is similar to that of Fig. 8c, indicating the presence of stable subharmonics.

The chaotic behavior in the liquid filled ultrasonic resonant system is generated at a second threshold (it was pointed out earlier that subharmonic generation requires a threshold power input). It is significant to notice from Fig. $9 \mathrm{~b}$ that at additional power level the chaos reverses 


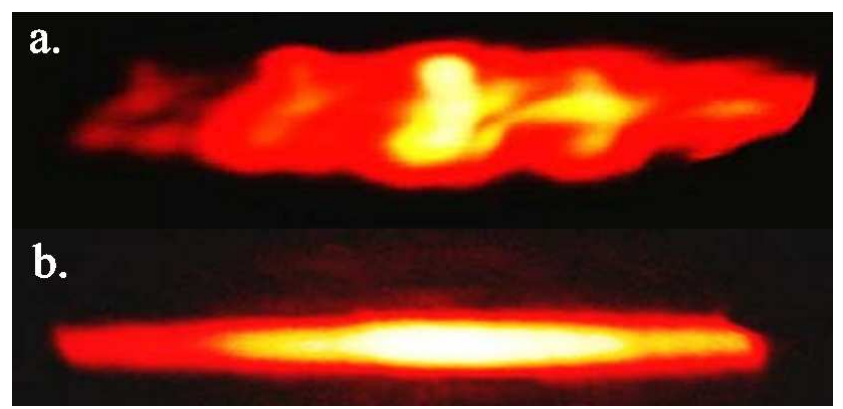

Fig. 9. Laser beam diffraction ultrasonic waves: (a) in the chaotic region $(450 \mathrm{~V})$, (b) beyond chaotic region $(500 \mathrm{~V})$.

into stable oscillation i.e. beyond chaos. This observation is quite rare in chaotic systems although some theoreticians are predicting the reversal of chaos. It should be mentioned that hysteresis is also observed when the power level changes from chaos to beyond and back to chaos. The question remains unanswered is the physical mechanism involved when chaos is produced. One possible mechanism is "turbulence" which may take place in the water at high power. Another possibility is phase transformation in water i.e. boiling and subsequent formation of bubbles. Both of these mechanisms are examples of chaotic behavior. Additional work is required to evaluate the source of chaos in this system.

\section{Summary}

It was demonstrated that diffraction of light through ultrasonic waves with finite amplitudes provides new observations and better understanding in nonlinear acoustics.

\section{References}

[1] P. Debye, F.W. Sears, Proc. Natl. Acad. Sci. 18, 409 (1932).

[2] R. Lucas, P.J. Biquard, J. Phys. Radium 194, 2132 (1932).

[3] C.V. Raman, N.S. Nath, Proc. Ind. Acad. Sci. 2, 406 (1935).

[4] K.L. Zankel, E.A. Hiedemann, J. Acoust. Soc. Am. 31, 1366 (1959).

[5] M.A. Breazeale, J. Acoust. Soc. Am. 33, 857 (1961).

[6] F. Fox, W. Wallace, J. Acoust. Soc. Am. 26, 994 (1959).

[7] E. Fubini-Giron, Alta Frequenza 4, 530 (1935).

[8] L.E. Hargrove, J. Acoust. Soc. Am. 34, 511 (1960).

[9] W. Keck, R.T. Beyer, Phys. Fluids 3, 346 (1960).

[10] L. Adler, E.A. Hiedemann, J. Acoust. Soc. Am. 34, 410 (1962).

[11] J.F. Eykman, Rec. Trav. Chim. 14, 177 (1895).

[12] V.A. Krasilnikov, V.V. Shklovskaya-Kordi, L.K. Zarembo, J. Acoust. Soc. Am. 29, 642 (1957).

[13] A. Korpel, R. Adler, Appl. Phys. Lett. 7, 106 (1965).

[14] L. Adler, M.A. Breazeale, Naturwissenschaften 8, 385 (1968).

[15] L. Adler, T. Yost, J. Cantrell, J. Acoust. Soc. Am. 133, 3555 (2013). 